\title{
RNA-Seq expression profiling of genes related to neurodegenerative disorders affecting the human retina
}

Laura Campello, José Martín-Nieto ${ }^{凶}$

Universidad de Alicante, Alicante, Spain

\section{Motivation and objectives}

Sight is likely the most important human sense. In this context, it is well known that human neurodegenerative diseases, such as Parkinson's disease (PD) and the neuromuscular disorders called dystroglycanopathies (DGPs), cause retinal impairments and consequently vision loss (Muntoni and Voit, 2004; Bodis-Wollner, 2009). We have characterized the expression of PD-related genes SNCA ( $\alpha$-synuclein), PARK 2 (parkin) and UCHL 1 in the mammalian retina (Martínez-Navarrete et al., 2007; Esteve-Rudd et al., 2010) and have found that a number of DGP-related genes are expressed in this tissue as well (Martín-Nieto et al., 2012). We have also described morphological (Cuenca et al., 2005) and proteomic (EsteveRudd et al., 2013) alterations taking place in the primate retina associated with parkinsonism. In this work we have attempted to catalog all known genes linked to PD and DGPs expressed in the human retina and quantify their mRNA levels. We have also focused in identifying transcript variants of these genes, in order to possibly correlate them with propensity to visual impairment.

\section{Methods}

Human retina reference RNA extracted from a pool of 29 Caucasian donors (both sexes, ages 20-60) was obtained from Clontech-BD. Total RNA was reverse-transcribed and amplified using the SMART PCR CDNA Synthesis kit (Clontech-BD). The obtained cDNA was mechanically cut into 100 bp fragments by ultrasonication, and a CDNA library was constructed using NEBNext reagents (New England Biolabs). There after, the cDNA was sequenced on an Illumina HiSeq 2000 system by Otogenetics Corp. using a read length of $100 \mathrm{bp}$, paired-end sequencing and a depth coverage of 100 million reads. Subsequent bioinformatic analyses of the obtained sequenceswere performed by Otogenetics and Genometra companies. The data processing protocol included the following computational tools:
- Sequence data quality control: FastQC software (http://www.bioinformatics.babraham. ac.uk/projects/fastac/)

- Sequence data files handling: Samtools (http://samtools.sourceforge.net/)

- Mapping: TopHat software (http://tophat. cbcb.umd.edu/), including the ultra highthroughput short read aligner Bowtie (http:// bowtie.cbcb.umd.edu).

- Transcript identification: Cufflinks (http://cufflinks.cbcb.umd.edu/).

- Expression level quantification: Cufflinks software and Qualimap platform (GarcíaAlcalde et al., 2012; http://qualimap.bioinfo. cipf.es/).

- Sequence data alignment visualization: Integrative Genome Viewer (IGV) (www.broadinstitute.org/igv/vl.4).

\section{Results and Discussion}

We have evidenced that most of the neurodegenerative disease-related genes assessed are expressed in the human retina, and their mRNA expression levels have been quantitated in terms of fragments per kilobase per million reads (FPKM) through RNA-Seq technology. These include the PD-linked genes SNCA, PARK2, UCHL1, DJ1 and PINK1, and the DGP-linked genes POMT1, POMT2, POMGNT1, FKTN (fukutin), FKRP and LARGE, among others. Besides, we have characterized the expression profile of such genes in the retina by determining their exonic, intronic and exon-intron junction expression levels. These data have allowed us to examine the alternative splicing pattern of particular genes, and as a result a number of new transcript variants have been identified. We are currently attempting to correlate particular splice variants with loss of gene function. We believe that this research should be of potential usefulness to understand the molecular bases of sight deficiencies associated with neurodegenerative disorders. 


\section{Acknowledgements}

This research has been supported by the Instituto de Salud Carlos III grant ref. PI09/1623 (†o J.M.-N.). L.C. was the recipient of a predoctoral contract from the Universidad de Alicante.

\section{References}

Bodis-Wollner I (2009) Retinopathy in Parkinson disease. J Neural Transm 116(11), 1493-1501. doi: 10.1007/s00702009-0292-z.

Cuenca N, Herrero M-T, Angulo A, De Juan E, MartínezNavarrete GC et al.(2005) Morphological impairments in retinal neurons of the scotopic visual pathway in a monkey model of Parkinson's disease. J Comp Neurol 493(2) 261-273.doi: 10.1002/cne.20761

Esteve-Rudd J, Campello L, Herrero M-T, Cuenca N, MartínNieto $J(2010)$ Expression in the mammalian retina of parkin and $\mathrm{UCH}-\mathrm{Ll}$, two components of the ubiquitinproteasome system. Brain Res 1352,70-82. doi: 10,1016/j. brainres. 2010.07.019.
Esteve-Rudd J, Campello L, Bru-Martínez R, FernándezVillalba E, Herrero MT, Cuenca N, Martín-Nieto J (2013) Alterations in energy metabolism, neuroprotection and visual signal transduction in the retina of parkinsonian, MPTP-treated, monkeys. PLoS One (submitted)

García-Alcalde F, Okonechnikov K, Carbonell J, Cruz LM, Götz S, Tarazona S, Dopazo J, Meyer TF, Conesa A (2012) Qualimap: evaluating next-generation sequencing alignment data. Bioinformatics 28(20), 2678-2679. doi: 10.1093/. bioinformatics/bts503

Martín-Nieto J, Uribe ML, Arenas CM, Rubio M, Aza M, Cruces J, Campello L (2012) All dystroglycanopathies-causing genes are expressed in the retina of adult mammals. FEBS J 279(Suppl. 1), 311

Martínez-Navarrete GC, Martín-Nieto J, Esteve-Rudd J, Angulo A, Cuenca N (2007) $\alpha$-Synuclein gene expression profile in the retina of vertebrates. Mol Vis 13, 949-961.

Muntoni F, Voit T (2004) The congenital muscular dystrophies in 2004: a century of exciting progress. Neuromusc Disord 14(10), 635-649. 